\title{
The effect of polymer chain length on the mechanical properties of triblock copolymer gels
}

\author{
Tanya L. Chantawansri ${ }^{*}$, T, Timothy W. Sirk ${ }^{*}, \ddagger$ \\ Randy Mrozek ${ }^{*}$, Joseph L. Lenhart ${ }^{*}$, Martin Kröger ${ }^{\dagger}$, Yelena R. Sliozberg ${ }^{*}$, \\ *US Army Research Laboratory, 4600 Deer Creek Loop, Aberdeen Proving Ground, MD 21005 \\ ${ }^{\dagger}$ Department of Materials, Polymer Physics, ETH Zürich, CH-8093 Zürich, Switzerland \\ ' Corresponding Author, email: tanya.chantawansri.civ@ mail.mil; Phone: 410-306-2777 \\ ${ }^{\ddagger}$ Current affiliation: TKC Global, 2100 Muir Way, Bel Air, MD 21015
}

\begin{abstract}
An ABA triblock copolymer in a midblock selective solvent is studied as a function of the chain length, using a novel dissipative particle dynamics (DPD) model which includes a modified segmental repulsive potential (mSRP) that restricts chain crossing. Deformation under uniaxial tension using the DPD method, with and without the mSRP, were used to extract the cross-link and entanglement portions of the modulus. The results exhibit the expected theoretical scaling with chain length for lower strain rates. Structural properties, such as bridge fraction and the extent of entanglements in the polymer matrix, were also quantified.
\end{abstract}

Keywords: polymer mechanics, coarse-grained modeling, block copolymer gels

\section{Introduction}

There is substantial interest in thermoplastic elastomeric gels (TPE), which utilize block copolymer phase separation to incorporate physical cross-links into the polymer matrix. The polymer used in TPE gels consists of two or more chemically dissimilar polymer chains covalently bound together to form a large macromolecule, where immiscibility between the distinct species in the polymer and solvent, and their molecular weights, dictate the self assembly of nanostructures. The observed nanostructure affects the number of physical cross-links arising due to bridges that form between microdomains [1-3]. For polymers of high molecular weight, 
bridges and entanglement constraints (such as loops, kinks, and knots [4]) hinder the motion of the polymer. Of particular interest in experimental studies are poly[styrene-b-(ethylene-cobutylene)-b-styrene] (SEBS) based gels which are the hardest and most resilient to degradation.

Numerous experimental studies have been performed on SEBS gels swollen with mineral oil. Kim et al. [5, 6] studied the effect of concentration of different hydrocarbon oils on the linear viscoelastic behavior, thermal transitions, swelling behavior and gel properties associated with physical gelation. The effect of mineral oil concentration (70 to $90 \mathrm{wt} \%$ ), temperature, and SEBS molecular weight on morphology and mechanical properties was studied by Laurer et al. [7-9]. The correspondence between rheology and morphology to temperature was also explored for SEBS gels at two different molecular weights by Sugimoto et al. [10]. Additives to SEBS TPEGs such as nanofillers, homopolymer, and resins were also investigated, where their effect on morphology in addition to thermo-mechanical and bulk properties were explored [10].

Computational studies have also been performed on systems similar to SEBS and mineral oil TPEG gels, where the polymer is typically modeled as an ABA-type triblock copolymer in a B-selective solvent. Chantawansri et al. [11], computed a phase diagram of these gels as a function of solvent loading, molecular weight, and interaction parameters using dynamic density functional theory for comparison with available experimental data. Grand canonical Monte Carlo was used by Kim et al. [12] to study the shape, size, distribution, and internal structures of micelles at high solvent volume fractions, while Sliozberg et al. $[13,14]$ used dissipative particle dynamics (DPD) to study the morphology of the unentangled gels composed of triblock and diblock polymers and the mechanical properties of triblock gels. Shull and coworkers [15] used the unit cell approximation in self-consistent field theory (SCFT) to study the effect of triblock copolymer midblock length and temperature on the micelle size, geometry, aggregation number, 
bridging fraction, solvent osmotic pressure and form factor as a compliment to experimental data.

Despite the previous computational studies of polymer gels, a comprehensive understanding of the mechanical response of high molecular weight, topologically entangled, polymer gels is still lacking. Sliozberg et al. [16] explored the effect of entanglements between a high molecular weight solvent and a polymer network on the modulus using a generic coarsegrained bead spring model. They found that solvent entanglements dictate the time-dependent elastic modulus, and that a highly entangled solvent can be used to adjust the rate dependent modulus in these systems. For phase-separating gels, the DPD method was used with a modified segmental repulsive potential (mSRP) to study the structural and mechanical properties of entangled gels under deformation as a function of polymer volume fraction [17]. The mSRP was used to prevent unphysical chain crossing which typically occurs due to the soft repulsive potentials in standard DPD [18]. The DPD-mSRP approach was used to qualitatively reproduce the theoretical scaling of the entanglement and cross-link portion of the elastic modulus with polymer concentration, while maintaining the original structure of the gel obtained using DPD.

In this work, we used the DPD-mSRP approach to study the structural and mechanical properties of a specific composition of SEBS gels under deformation for various polymer chain lengths, from unentangled to entangled. The chain length of the polymer, $N_{T}$, and effectively the midblock, $N$, were varied to determine the effect of entanglements in the solvated EB polymer matrix on bridge fraction and the cross-link and entanglement portions of the elastic modulus.

\section{Model System}


We consider a poly[styrene- $b$-ethylene-co-butylene- $b$-styrene] (SEBS) triblock copolymer in mineral oil, where the mineral oil is selective to the EB midblock [11]. In particular, we are interested in the composition of SEBS produced by Kraton [19] labeled SEBS G1652, which is 33 vol \% styrene. The SEBS will be diluted to a volume fraction of 0.325 which corresponds to the micellar/spherical phase [11] and is of interest in the development of tissue stimulants for body armor certification. To model this system we approximate the polymer as an ABA-type triblock copolymer immersed in a midblock selective solvent. The crosslink and entanglement contributions to the elastic modulus as well as the bridge fraction were found using the DPD method.

In DPD, particles interact through pairwise, two-body, short-ranged forces that include contributions from conservative, dissipative, and random forces. The conservative force is taken as the sum of a bonded force acting between topologically connected monomers and a pairwise non-bonded force, $\mathrm{F}_{\mathrm{c}}=a_{\mathrm{ij}}\left(1-r / r_{c}\right)$, acting between particles of type $i$ and $j$ separated by a distance $r$ and truncated at $r_{c}$. The repulsive strength parameter $a_{\mathrm{ij}}$ for unlike-particles $(i \neq j)$ is related to the Flory-Huggins parameter $\chi$ as $\chi=0.231\left(a_{i j}-a_{i i}\right)$ [20]. For the remainder of the manuscript, DPD parameters will be in reduced units of length, energy, time and mass, which are $r_{c}, k_{B} T$, $\tau=r_{c} \sqrt{m / k_{B} T}$, and $m$, respectively.

A modified segmental repulsive potential (mSRP) was added to DPD to restrict the unphysical crossing of polymer chains. The mSRP is a softly repulsive potential that acts between pairs of bonded beads. By applying mSRP and an angle bending potential with parameters described by Sirk et al. [18], reptation behavior can be observed for chains over 15 beads in length without disturbing the chain structure and thermodynamic properties of the standard DPD method. The DPD parameters specific to the ABA triblock gel can be found in 
Chantawansri et al. [17]. A more comprehensive description of DPD method and mSRP can be found elsewhere [17, 18, 20, 22].

Simulations were performed on the molecular dynamics simulator LAMMPS [23, 24] for a total of three replicas for system sizes of 1.15 to 2.15 million atoms. The polymers and solvent are discretized using coarse grained particles of equal volume, where the end and mid blocks of the polymer are composed of beads of type "A" and "B", respectively, while the solvent is composed of two beads of type "S". Equilibrated structures were generated by first assigning chain structures according to a non-reversal-random-walk, then performing DPD using a soft potential for non-bonded interactions. Initially, DPD simulations were performed with $\Delta a=a_{\mathrm{ij}}$ $a_{\mathrm{ii}}=0 \mathrm{k}_{\mathrm{B}} \mathrm{T}$, to remove artificial strain in the system after the generation process for $10^{5}$ steps using a time step of 0.01 . The values of $\Delta a$ between the different bead pairs were then increased to values shown in Table 1 to obtain phase separation. Since the effect of polymer chain length is of interest, the values of $\Delta a$ were chosen such that $\Delta a N_{T}$ (and $\chi N_{T}$ ) were constant for all chain lengths. To maintain numerical stability during the initial stages of phase separation, DPD was performed for $5 \cdot 10^{6}$ steps with a time step of 0.01 . Equilibration of the DPD system is then performed with a time step of 0.04 for $2.5 \cdot 10^{7}$ steps, followed by DPD-mSRP simulations for $1 \cdot 10^{6}$ time steps with a time step of 0.01 .

Table 1. $\Delta a$ parameters used for each discretization of the triblock copolymer, where $a_{\mathrm{ii}}=60 \mathrm{k}_{\mathrm{B}} \mathrm{T}$.

\begin{tabular}{|c|c|c|}
\hline \multirow{2}{*}{ Chain length } & \multicolumn{2}{|c|}{$\Delta a\left(\mathrm{k}_{\mathrm{B}} \mathrm{T}\right)$} \\
\cline { 2 - 3 } & $\mathrm{A}-\mathrm{B}$ & $\mathrm{A}-\mathrm{S}$ \\
\hline $\mathrm{A}_{10} \mathrm{~B}_{40} \mathrm{~A}_{10}$ & 80 & 275 \\
\hline $\mathrm{A}_{20} \mathrm{~B}_{80} \mathrm{~A}_{20}$ & 40 & 137.5 \\
\hline $\mathrm{A}_{30} \mathrm{~B}_{120} \mathrm{~A}_{30}$ & 27 & 91.7 \\
\hline $\mathrm{A}_{40} \mathrm{~B}_{160} \mathrm{~A}_{40}$ & 20 & 68.8 \\
\hline $\mathrm{A}_{50} \mathrm{~B}_{200} \mathrm{~A}_{50}$ & 16 & 55 \\
\hline $\mathrm{A}_{60} \mathrm{~B}_{240} \mathrm{~A}_{60}$ & 13.3 & 45.8 \\
\hline $\mathrm{A}_{70} \mathrm{~B}_{280} \mathrm{~A}_{70}$ & 11.4 & 39.3 \\
\hline
\end{tabular}


After equilibration of the DPD and DPD-mSRP systems, both were subjected to uniaxial tensile affine deformations in the $z$-direction under constant volume conditions with a time step of 0.0075 . Two constant true strain rates of $\dot{\varepsilon}=10^{-4}$ and $10^{-3}$ were considered. The elongational stress, $\sigma$, was then calculated from the difference of the axial and lateral normal stresses. The results were averaged over three replicas unless otherwise stated.

\section{Results and Discussion}

After construction and equilibration of the systems using the procedure described in the previous section, the midblocks in the polymer chain were analyzed using the $\mathrm{Z} 1$ code $[25,26]$ to estimate the extent of entanglements as a function of midblock length. The $\mathrm{Z} 1$ code reduces the contour lengths of the chains monotonically through geometrical moves in the limit of zero primitive chain thickness to extract the number of interior "kinks" $(Z)$ in the three-dimensional primitive path of each chain $[26,27]$. This calculation assumes that the entanglement contribution of the end blocks is negligible. From $\langle Z\rangle$, the entanglement length for the gel, $N_{e}^{\text {gel }}$, can be extracted using a variety of estimators, which determine $N_{e}^{\text {gel }}$ based on results from a single chain (S-estimators) or multiple chains (M-estimators). The notation $N_{e}^{\text {gel }}$ is used to distinguish between the entanglement molecular weight of the gel and the melt $\left(N_{e}\right)$, where $N_{e}^{\text {gel }}$ $<N_{e}$. We use M-estimators since they are shown to converge for marginally entangled systems $\left(N \leq N_{e}{ }^{\text {gel }}\right)$ such as those studied here. To estimate $N_{e}^{\text {gel }}$, based solely on kinks, we used the approximate M-kink [28]. In this approximation, $N_{e}$ gel is estimated as the inverse of the coefficient in the linear regression of $\langle Z\rangle$ and $N$, where $\lim _{N \rightarrow \infty} N_{e}^{\text {gel }}(N)=N_{e}^{\text {gel }}$. A plot of $\langle Z\rangle$ as a 
function of $N$ is shown in Figure 1. $N_{e}^{\text {gel }}(N)$ diverges as $N \rightarrow 0$, thus we have excluded midblocks of small molecular weight $(\mathrm{N}<=40)$. Fitting the data we obtained $\langle Z\rangle=0.01092 N+0.4090$, where the reciprocal of the slope gives an estimate of $N_{e}{ }^{\text {gel }}=91.6$. Although this estimate of $N_{e}$ gel is only rigorously correct in the limit of $N \rightarrow \infty$, it can nonetheless be used to quantify the extent of entanglements in our systems, where we can calculate the number of entanglements in our gel system using $n_{e} e^{\text {gel }}=N / N_{e}{ }^{\text {gel }}$. When $N$ becomes larger than $N_{e}{ }^{\text {gel }}$, or $n_{e}{ }^{\text {gel }}>1$, the effect of entanglements becomes important. From Table 2, we observe that entanglement effects should be seen for $N$ between 80 to 120 for this solvated system.

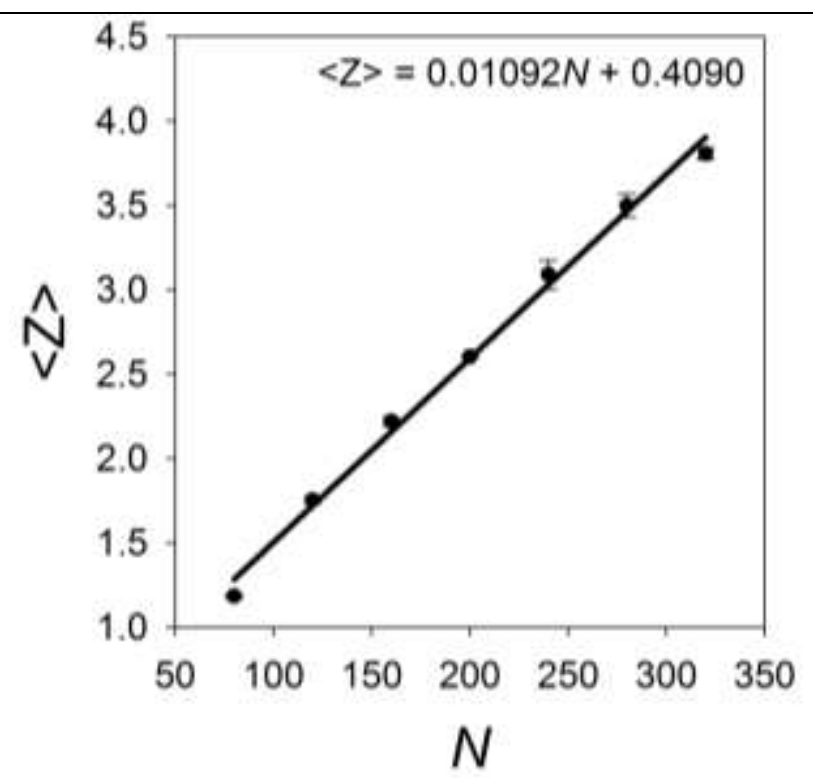

Figure 1. The average interior "kinks", $\langle Z\rangle$, as a function of the number of beads, $N$, in the midblock.

Table 2. Number of entanglements in melt and gel for various chain lengths.

\begin{tabular}{|c|c|c|c|}
\hline Polymer & $N$ & $\langle Z\rangle$ & $n_{e}^{\text {gel }}$ (M-kink) \\
\hline
\end{tabular}




\begin{tabular}{|c|c|c|c|}
\hline $\mathrm{A}_{10} \mathrm{~B}_{40} \mathrm{~A}_{10}$ & 40 & $0.512 \pm 0.0024$ & 0.44 \\
\hline $\mathrm{A}_{20} \mathrm{~B}_{80} \mathrm{~A}_{20}$ & 80 & $1.183 \pm 0.0073$ & 0.87 \\
\hline $\mathrm{A}_{30} \mathrm{~B}_{120} \mathrm{~A}_{30}$ & 120 & $1.752 \pm 0.016$ & 1.31 \\
\hline $\mathrm{A}_{40} \mathrm{~B}_{160} \mathrm{~A}_{40}$ & 160 & $2.218 \pm 0.0267$ & 1.75 \\
\hline $\mathrm{A}_{50} \mathrm{~B}_{200} \mathrm{~A}_{50}$ & 200 & $2.601 \pm 0.0018$ & 2.18 \\
\hline $\mathrm{A}_{60} \mathrm{~B}_{240} \mathrm{~A}_{60}$ & 240 & $3.088 \pm 0.086$ & 2.62 \\
\hline $\mathrm{A}_{70} \mathrm{~B}_{280} \mathrm{~A}_{70}$ & 280 & $3.500 \pm 0.069$ & 3.05 \\
\hline $\mathrm{A}_{80} \mathrm{~B}_{320} \mathrm{~A}_{80}$ & 320 & $3.804 \pm 0.036$ & 3.50 \\
\hline
\end{tabular}

The largest polymer chain length that we consider $\left(\mathrm{A}_{80} \mathrm{~B}_{320} \mathrm{~A}_{80}\right)$ corresponds to approximately 3.5 entanglements when solvated with a B selective solvent at a polymer volume fraction of 0.325 . Increasing the number of entanglements is beyond our current computational capabilities due to the longer relaxation time and larger box size associated with increasing the chain length. Even with this modest amount of entanglements, there are pronounced differences

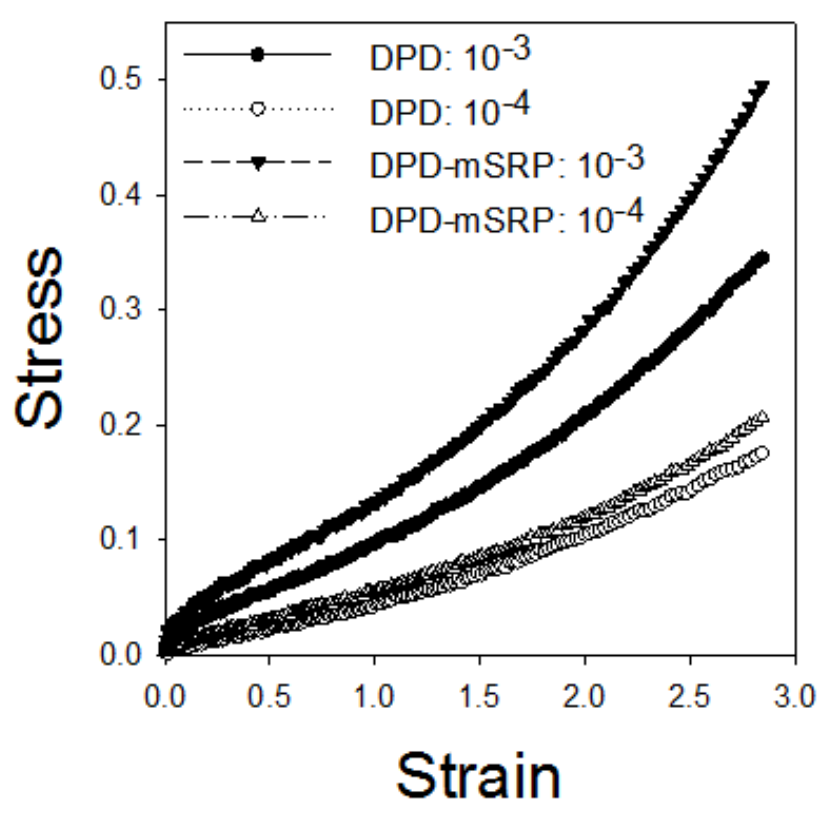

Figure 2. Stress-strain curves for $\mathbf{A}_{80} \mathbf{B}_{320} \mathbf{A}_{80}$ for strain rates of $\dot{e}=10^{-4}, 10^{-3}$. 
in the stress-strain behavior obtained using DPD and DPD-mSRP when the chains are sufficiently entangled. Figure 2 shows the stress strain curves for $A_{80} B_{320} A_{80}$ for strain rates of $\dot{\varepsilon}=10^{-4}$ and $10^{-3}$. Values of $\langle Z\rangle$ vary slightly during deformation for strains up to 1.0, where we observe a decrease of $2 \%$ and $5 \%$ at $\dot{\varepsilon}=10^{-4}$ and $10^{-3}$, respectively, which agrees with published findings [29].We will only consider strains up to 1.0 in subsequent calculations.

Due to the additional entanglement contribution to the stress, stress obtained through the DPD-mSRP is higher than that obtained using DPD alone, where the difference in the curves increases with the number of entanglements. In addition, the difference between the two curves increases with increasing strain rates since entanglement effects become more pronounced for higher strain rates [16]. From these stress-strain curves, we can extract two contributions to the stress $(\sigma)$. The stress is composed of a cross-link contribution $\left(\sigma_{\mathrm{c}}\right)$ associated with bridges and an entanglement contribution $\left(\sigma_{\mathrm{e}}\right)$ from the midblock in solvent. The cross-link contribution to the modulus can be approximated as:

$$
G_{c}=k_{B} T \phi_{B}\left[\frac{C_{B}}{N}\right]
$$

where $\mathrm{k}_{\mathrm{B}}$ is the Boltzmann constant, $T$ is the temperature, $\phi_{\mathrm{B}}$ is the bridge fraction, and $\mathrm{C}_{\mathrm{B}}$ is the number concentration of the triblock specific B beads [4]. For the micellar morphology, a bridge occurs when the end block of the triblock resides in two different micelles, while a loop occurs when both ends of the triblock reside in the same micelle. Since we do not observe any free ends, the bridge fraction is defined as the number of bridges divided by the total number of bridges and loops. The bridge fraction was calculated before and after the addition of the mSRP, where we observed no difference in the bridge fraction. The introduction of mSRP has also been shown to retain structural quantities of the system obtained after DPD simulations such as the morphology, 
number of micelles, aggregation number, and the bridge fraction, while only minimally affect the size of the micelles (less than 3\%) [17]. The bridge fraction was calculated for our system as a function of different chain lengths. As shown in Figure 3, we observe that the bridge fraction converges to $\sim 0.6$. For this system, bridge fraction depends on the relative size of the midblock with respect to the endblocks and the polymer concentration, but it does not depend on chain length [14, 30-32]. Instead, chain length affects the dynamics of the system where entanglements slow down local mobility of the polymer chains [4].

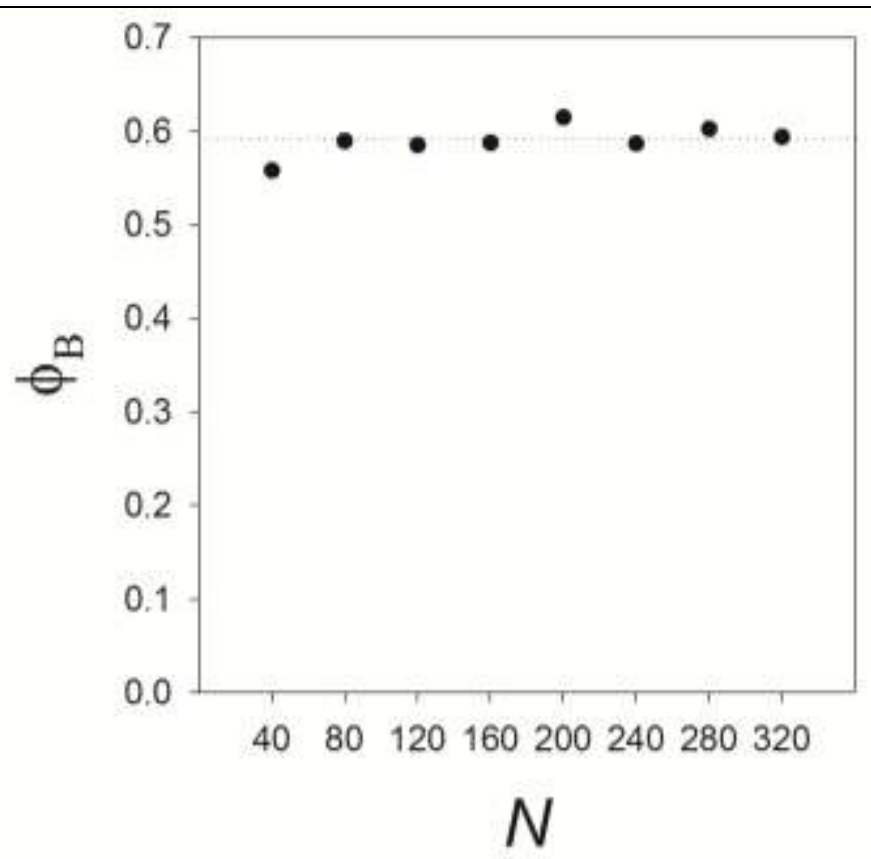

Figure 3. Plot of the bridge fraction, $\phi_{\mathrm{B}}$, as a function of the number of beads, $N$, in the midblock. A dotted line is shown at the average $\phi_{B}=0.59$ to guide the eye.

Due to the use of soft potentials, we take the stress obtained using DPD as having only a contribution due to cross-links, while the addition of mSRP would capture the contributions of both cross-links and entanglements for sufficiently long polymers. If the stress responses for 
cross-links and entanglements are independent and additive, the elastic modulus can be separated into corresponding cross-link and entanglement terms. The cross-link portion of the modulus can be extracted from the stress using the theory of rubber elasticity [4] given by:

$$
\sigma=G_{c}\left(\lambda^{2}-\frac{1}{\lambda}\right)
$$

where $G_{c}$ is the cross-link portion of the modulus and $\lambda$ is the stretch ratio. Fitting Eq. 2 to the stress calculated using the DPD method for strains between 0 and 1 , values of $\mathrm{G}_{\mathrm{c}}$ were extracted for all $N$, and are shown in Figure 4 for strain rates of $\dot{\varepsilon}=10^{-4}$ and $10^{-3}$. Eq. 1 predicts $\mathrm{G}_{\mathrm{c}} \sim 1 / N$ since $\mathrm{k}_{\mathrm{B}} \mathrm{T} \phi_{\mathrm{B}} \mathrm{C}_{\mathrm{B}}$ is effectively constant for our system. To test this trend, curves of $\mathrm{G}_{\mathrm{c}} \sim 1 / N$ are also shown in Figure 4, where the plot was normalized such that $\mathrm{G}_{\mathrm{c}}$ for $N=40$ is equal to the value extracted from the stress curve. From the plot, $\mathrm{G}_{\mathrm{c}}$ for $\dot{\varepsilon}=10^{-4}$ follows the correct $1 / \mathrm{N}$ trend but the plot deviates at the higher strain rate of $\dot{\varepsilon}=10^{-3}$. At high strain rate Eq. 2 does not hold, possibly due to an increased importance of viscous (inelastic) contributions to the stress. We note that the absolute value of $\mathrm{G}_{\mathrm{c}}$ tends to be overestimated through by Eq. 2 [33], and we therefore regard Eq. 2 as useful only for obtaining qualitative trends. 


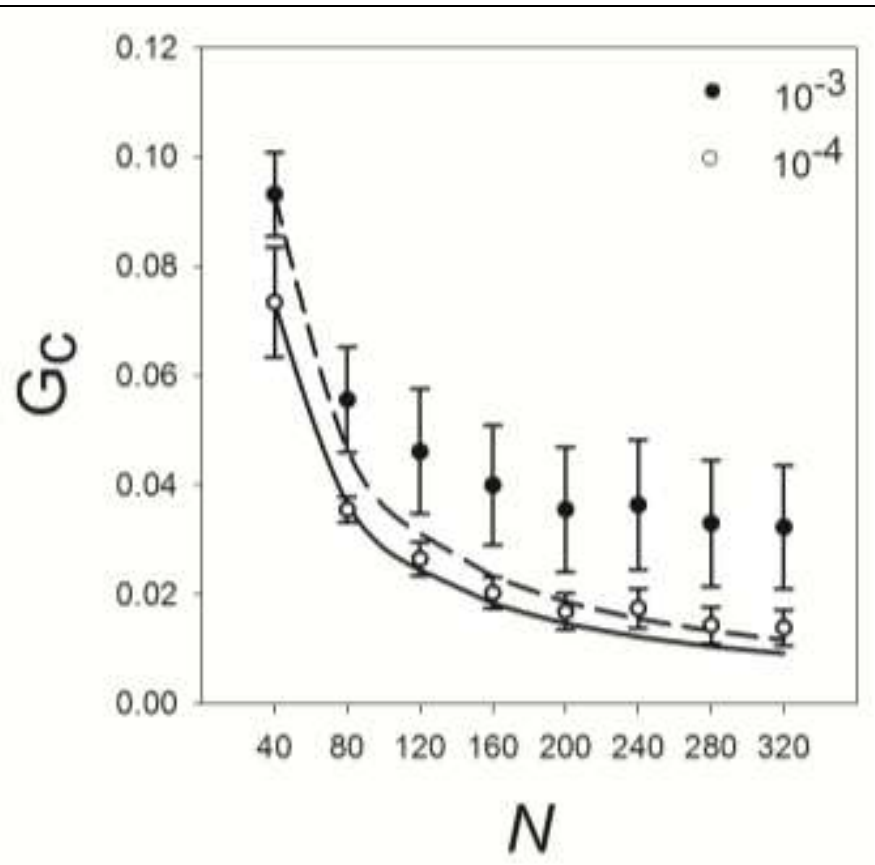

Figure 4. Plot of cross-link contribution to the modulus, $G_{c}$, as a function of the number of beads, $N$, in the midblock.

The entanglement portion of the modulus can be extracted from the stress by using the "Slip-Tube Model" of Rubinstein and Panyukov [34] for entangled networks:

$$
\sigma=\left(G_{c}+\frac{G_{e}}{0.74 \lambda+0.61 \lambda^{-1 / 2}-0.35}\right)\left(\lambda^{2}-\frac{1}{\lambda}\right),(3)
$$

where $G_{e}$ is the entanglement portion of the elastic modulus. For unentangled networks $\left(G_{e}=0\right)$, Eq. 3 reduces to Eq. 2. The stress obtained through DPD has only the cross-link contribution, $\sigma_{\mathrm{DPD}}=\sigma_{\mathrm{c}}$, while the stress from DPD-mSRP has both contributions from cross-links and entanglements, $\sigma_{\mathrm{DPD}-\mathrm{mSRP}}=\sigma_{\mathrm{c}}+\sigma_{\mathrm{e}}$. Subtracting the two stresses allows the entanglement portion of the stress to be extracted. In this manner, $\mathrm{G}_{\mathrm{e}}$ was extracted by fitting the calculated $\sigma_{\mathrm{DPD}-\mathrm{mSRP}}$ $\sigma_{\text {DPD }}$ to Eq. 3 with $\mathrm{G}_{\mathrm{c}}=0$. Figure 5 shows $\mathrm{G}_{\mathrm{e}}$ values obtained for $N>N_{e}{ }^{\text {gel }}$ for strain rates of $\dot{\varepsilon}=10^{-}$ ${ }^{4}$ and $10^{-3}$. Values of $N<N_{e}$ gel are omitted since they have less than one entanglement. All numerical fits were performed up to the onset of strain hardening (taken here as a strain of 1.0). 
Although the difference of the stress obtained using DPD and DPD-mSRP were noisy due to the high solvation of the system, a trend can be discerned in $\mathrm{G}_{\mathrm{e}}$ with $N$, where $\mathrm{G}_{\mathrm{e}}$ increases slightly and plateaus. Assuming that cross-links achieved through the formation of micelles are fixed and move in an affine manner, $\mathrm{G}_{\mathrm{e}} \sim \mathrm{k}_{\mathrm{B}} \mathrm{T} \mathrm{C}_{\mathrm{B}}\left(N_{e}^{\text {gel }}\right)^{-1}$ when $N \gg N_{e}^{\text {gel }}$ [4]. Since $\mathrm{C}_{\mathrm{B}}$ and $N_{e}^{\text {gel }}$ are constant for our systems, $\mathrm{G}_{\mathrm{e}}$ should converge once the polymer is sufficiently entangled.

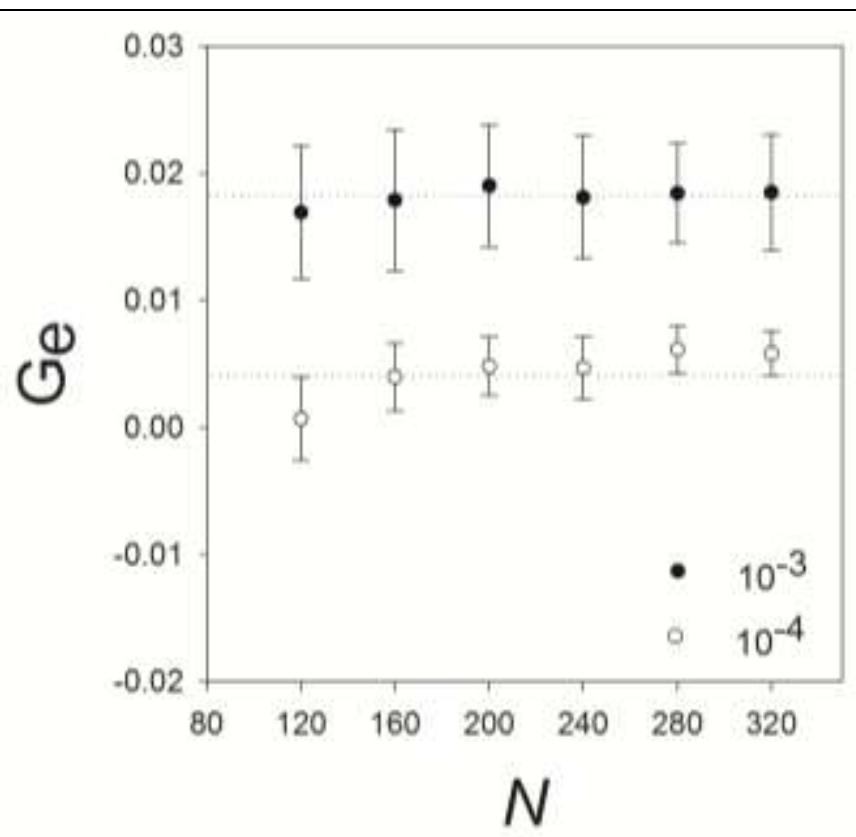

Figure 5. Plot of entanglement contribution to the modulus, $G_{e}$, as a function of the number of beads, $N$, in the midblock. A dotted line is shown at the average $G_{e}=0.018$ $\left(\dot{\varepsilon}=10^{-3}\right)$ and $0.004\left(\dot{\varepsilon}=10^{-4}\right)$ to guide the eye.

\section{Conclusion}

We have used the dissipative particle dynamics (DPD) with and without the modified segmental repulsive potential (mSRP) to calculate the bridge fraction as well as the cross-link and entanglement portions of the elastic modulus for an ABA triblock copolymer gel. For a gel composed of $33 \%$ A within a B-selective solvent at a polymer content of $32.5 \%$, we obtained a 
bridge fraction of approximately 0.6 for various chain lengths, where the extent of entanglements formed in the polymer matrix composed of the midblock and solvent were calculated using the $\mathrm{Z} 1$ code. In addition, deformation curves under uniaxial tension were calculated for strain rates of $\dot{\varepsilon}=10^{-4}$ and $10^{-3}$, where we extracted both the cross-link and entanglement portion of the elastic modulus from the stress as a function of the midblock length, $N$, by assuming that the contributions from cross-links, $G_{c}$, and entanglements, $G_{e}$, are independent and additive. $G_{c}$ was shown to exhibit the theoretical $\sim 1 / N$ behavior at the lower strain rate while deviations occur at the higher strain rate. $\mathrm{G}_{\mathrm{e}}$ was shown to plateau as a function of $N$ for sufficiently entangled systems, as would be expected from theoretical models.

\section{Acknowledgements}

TWS was supported in part by an appointment to the Postgraduate Research Participation Program at the U.S. Army Research Laboratory administered by the Oak Ridge Institute for Science and Education through an interagency agreement between the U.S. Department of Energy and USARL. Calculations were performed on DOD High Performance Computing site at the AFRL through the Challenge Project C5M.

\section{References}

1. $\quad$ Erk, K.A. and K.R. Shull, Rate-dependent stiffening and strain localization in physically associating solutions. Macromolecules, 2011. 44: p. 932.

2. $\quad$ Seitz, M.E., et al., Self-assembly and stress relaxation in acrylic triblock copolymer gels. Macromolecules, 2007. 40(4): p. 1218.

3. Pham, Q.T., et al., Micellar solutions of associative triblock copolymers: The relationship between structure and rheology. Macromolecules, 1999. 32: p. 5139.

4. Rubinstein, M. and R.H. Colby, Polymer Physics. 2003, New York: Oxford University Press.

5. Kim, J.K., M.A. Paglicawan, and M. Balasubramanian, Viscoelastic and Gelation Studies of SEBS Thermoplastic Elastomer in Different Hydrocarbon Oils. Macromol. Res., 2006. 14(3): p. 365. 
6. Kim, J.K., et al., Influence of Hydrocarbon Oils on the Physical Gelation of Poly(styreneb-(ethylene-co-butylene)-b-styrene) (SEBS) Triblock Copolymers. Journal of Elastomers and Plastics, 2007. 39: p. 133.

7. Laurer, J.H., R. Bukovnik, and R.J. Spontak, Morphological Characteristics of SEBS Thermoplastic Elastomer Gels. Macromolecules, 1996. 29: p. 5760.

8. Laurer, J.H., et al., Thermoplastic elastomer Gels. I. Effects of Composition and Processing on Morphology and Gel Behavior. J. Polym. Sci., Part B: Polym. Phys., 1998. 36: p. 2379.

9. $\quad$ Laurer, J.H., et al., Thermoplastic Elastomer Gels. II. Effect of Composition and Temperature on Morphology and Gel Rheology. J. Polym. Sci., Part B: Polym. Phys., 1998. 36: p. 2513.

10. Sugimoto, M., et al., Rheology and Morphology Change with Temperature of SEBS/Hydrocarbon Oil Blends. J. Polym. Sci., Part B: Polym. Phys., 2009. 47(10): p. 955.

11. Chantawansri, T., et al., Phase behavior of SEBS triblock copolymer gels. Journal of Polymer Science Part B: Polymer Physics, 2011. 49(20): p. 1479.

12. Kim, S.H. and W.H. Jo, A Monte Carlo Simulation for Micellization of ABA- and BABtype triblock copolymers in selective solvents. Macromolecules, 2001. 34: p. 7210.

13. Sliozberg, Y.R., et al., Computational and experimental investigation of morphology in thermoplastic elastomer gels composed of AB/ABA blends in B-selective solvent. Soft Matter, 2011. 7: p. 7539.

14. Sliozberg, Y.R., et al., Modeling Viscoelastic Properties of Triblock copolymers: A DPD Simulation Study. J. Polym. Sci., Part B: Polym. Phys., 2009. 48: p. 15.

15. Bras, R.E. and K.R. Shull, Self-Consistent Field Theory of Gelation is Triblock Copolymer Solution. Macromolecules, 2009. 42(21): p. 8513.

16. Sliozberg, Y.R., et al., Effect of polymer solvent on the mechanical properties of entangled polymer gels: Coarse-grained molecular simulation. Polymer, 2013. 54: p. 2555.

17. Chantawansri, T., T. Sirk, and Y.R. Sliozberg, Entangled triblock copolymer gel: Morphology and Mechanical Properties. J. Chem. Phys., 2013. 138: p. 024908.

18. Sirk, T., et al., An enhanced entangled polymer model for dissipative particle dynamics. The Journal of Chemical Physics, 2012. 136: p. 134903.

19. Kraton Chemical: http://www.kraton.com/.

20. Groot, R.D. and P.B. Warren, Dissipative particle dynamics: Bridging the gap between atomistic and mesoscopic simulation. Journal of chemical physics, 1997. 107(11): p. 4423.

21. Kumar, S. and R. Larson, Brownian dynamics simulations of flexible polymers with spring-spring repulsions. The Journal of Chemical Physics, 2001. 114(15): p. 6937.

22. Español, P. and P.B. Warren, Statistical Mechanics of Dissipative Particle Dynamics. Europhysics Letters, 1995. 30(4): p. 191.

23. http://lammps.sandia.gov.

24. Plimpton, S.J., Fast parallel algorithms for short-range molecular dynamics. Journal of computational physics, 1995. 117: p. 1.

25. http://www.complexfluids.ethz.ch/Z1. 
26. Shanbhag, S. and M. Kröger, Primitive path networks generated by annealing and geometrical methods: Insights into differences. Macromolecules, 2007. 40: p. 2897.

27. Kröger, M., Shortest multiple disconnected path for the analysis of entanglements in twoand three-dimensional polymeric systems. Compt. Phys. Commun, 2005. 168: p. 209.

28. Hoy, R.S., K. Foteinopoulou, and M. Kröger, Topological analysis of polymeric melts: Chain length effects and fast-converging estimators for entanglement length. Phys. Rev. E, 2009. 80(3): p. 031803.

29. Andreev, M., et al., Approximations of the discrete slip-link model and their effect on nonlinear rheology predictions. J. Rheol., 2013. 57: p. 535.

30. Shen, W., J.A. Kornfield, and D.A. Tirrell, Structure and mechanical properties of artificial protein hydrogels assembled through aggregation of leucine zipper peptide domains. Soft Matter, 2007. 3: p. 99.

31. Szczubialka, K., K. Ishikawa, and Y. Morishima, Associating behavior of sulfonated polyisoprene block copolymers with short polystyrene blocks at both chain ends. Langmuir, 2000. 16(5): p. 2083.

32. Watanabe, H., T. Sato, and K. Osaki, Concentration dependence of loop fraction in styrene-isoprene-styrene triblock copolymer solutions and corresponding changes in equilibrium elasticity. Macromolecules, 2000. 33(7): p. 2545.

33. Grest, G.S., et al., Stress-strain relation of entangled polymer networks. J. Non-Cryst. Solids, 2000. 274: p. 139.

34. Rubinstein, M. and S. Panyukov, Elasticity of polymer networks. Macromolecules, 2002. 35: p. 6670. 
Graphical Abstract

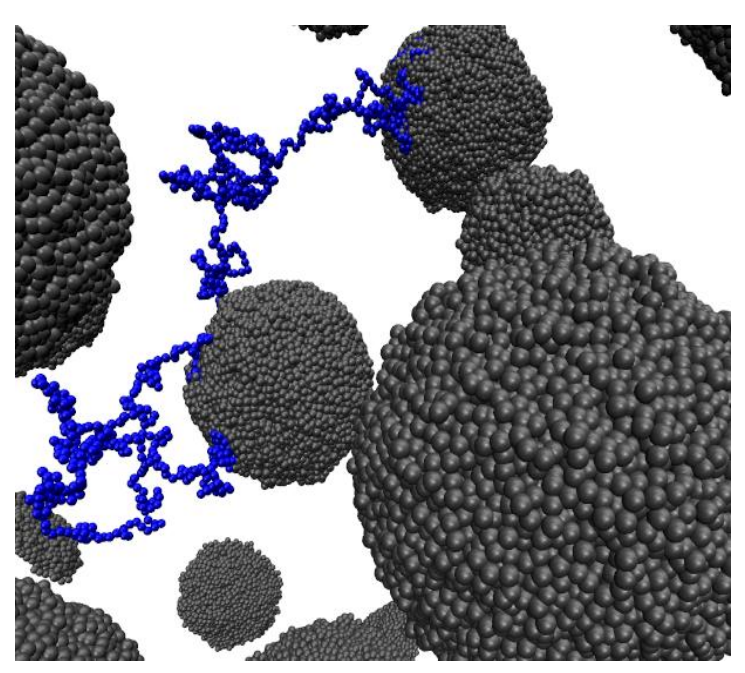

